\title{
Du portfolio au dossier professionnel : éléments de réflexion
}

\section{Dominique Bucheton}

\section{(2) OpenEdition}

1 Journals

\section{Édition électronique}

URL : https://journals.openedition.org/trema/1380

DOI : $10.4000 /$ trema.1380

ISSN : 2107-0997

\section{Éditeur}

Faculté d'Éducation de l'université de Montpellier

\section{Édition imprimée}

Date de publication : 1 janvier 2003

Pagination : 43-53

ISSN : 1167-315X

\section{Référence électronique}

Dominique Bucheton, «Du portfolio au dossier professionnel : éléments de réflexion », Tréma [En ligne], 20-21 | 2003, mis en ligne le 01 avril 2003, consulté le 21 septembre 2021. URL : http://

journals.openedition.org/trema/1380; DOI : https://doi.org/10.4000/trema.1380

Ce document a été généré automatiquement le 21 septembre 2021.

Trema 


\title{
Du portfolio au dossier professionnel : éléments de réflexion
}

\author{
Dominique Bucheton
}

1 La pratique du portfolio comme dispositif de formation entre dans sa quatrième année. Mis en place d'abord dans le module français à Perpignan par une équipe d'enseignants chercheurs - formateurs'intéressés par « l'écriture réflexive ${ }^{2}$ », puis essayé dans quatre groupes de $\mathrm{FGC}^{3}$ (formation générale et commune) PLC2, à Montpellier, il se généralise cette année sur l'ensemble de 1TUFM et se transforme en "Dossier professionnel ». À chacune de ses mises en pratique comme instrument de formation (appropriation de savoirs, régulation, évaluation formative et sommative), dans des contextes de formation ou dans des modules différents, il s'est modifié, adapté. On voudrait ici non faire le bilan de ces usages divers, mais simplement repérer les principes théoriques qui sous-tendent cette pratique de plus en plus fréquente en formation et mettre en évidence l'intérêt mais aussi quelques unes des difficultés ou dérives auxquelles le portfolio peut donner lieu et qu'il convient de contrôler. On voudrait aussi souligner ici, par l'exemple du portfolio, le lien étroit qui peut exister à l'ITJFM entre objets de recherche et objets de formation.

\section{Petit historique sur le portfolio et ses objectifs}

2 Il est issu du monde professionnel et artistique. Dans son portfolio, un artiste présente un échantillonnage de ses œuvres, des commentaires, articles critiques qui les ont accompagnées. Il montre son parcours de créateur, montre les traces de son travail, de l'évolution de sa pensée créative.

3 Le portfolio apparaît dans le monde scolaire aux États Unis dans les années 1980 en réaction aux évaluations standardisées centrées sur des compétences supposées être repérées dans un produit fini. Il remplace aujourd'hui dans certains pays et écoles de culture anglo-saxonne une partie des épreuves du baccalauréat par exemple. Dans les pays européens, c'est dans le domaine de l'enseignement des langues étrangères qu'on le trouve le plus mis en place. 
C'est un objet qui appartient à son auteur et le représente. De ce fait il est souple, malléable, transformable : une chemise à feuillets plastifiés, fait fréquemment l'affaire.

"Ce sont des collections de travaux assemblés par les élèves et les enseignants dans le but d'examiner non seulement les réalisations réussies, mais aussi les efforts, l'amélioration, les processus, le rendement. À travers la réflexion sur les collections systématiques de travaux, les élèves et les enseignants peuvent travailler ensemble à comprendre les forces de l'élève, ses besoins et ses progrès " (Tierney, Center et Desai, 1991, cités par S. Vanhulle, thèse de doctorat).

Cet assemblage finalisé des travaux d'élèves implique la participation active de l'élève à la sélection des contenus, voire même à la définition des critères de sélection et d'appréciation. L'objectif principal est de pousser l'élève à s'approprier ses travaux en vue de construire activement son apprentissage (Paulson et Meyer, 1991).

\section{Le portfolio en formation : les présupposés théoriques qui le sous-tendent}

6 Si nous avons lancé cette expérimentation sur le portfolio non dans la classe mais comme instrument de la formation, c'est à partir d'un certain nombre de recherches ${ }^{4}$ et présupposés théoriques qui sous-tendent sa pratique et plus généralement celle de l'écriture en formation. Nous les présentons ici.

\subsection{Le portfolio, un instrument réflexif}

\subsubsection{La mise à distance de l'expérience par sa verbalisation}

7 Le portfolio cherche à répondre à un problème fondamental pour l'enseignant chargé de l'accompagnement et de la formation des stagiaires: comment faire s'approprier (comprendre, reconstruire, transférer sur d'autres situations, d'autres contextes, les savoirs enseignés, expérimentés, les discussions organisées) ? Comment faire en sorte que ce qui se passe en formation, à l'IUFM ou dans les discussions avec les tuteurs se transforme en outils pour penser la classe (en instruments psychologiques si on reprend la terminologie vygotskienne) et ne soit pas considéré par les stagiaires comme un simple "passage obligé » de leur année de formation, passage dont ils ne se sentent pas du tout les acteurs?

8 Le postulat est alors que l'écriture singulière peut jouer ce rôle de mise à distance de l'expérience ou de la compréhension immédiate des événements survenus. Elle poursuit plusieurs objectifs :

- il s'agit en effet d'une écriture au plus proche de la formation hors la présence des élèves (la reprise d'une analyse de pratique collective, le compte rendu d'une rencontre avec un intervenant RASED, CEFISEM, etc), comme de la formation en leur présence, dans l'action dans l'établissement (l'analyse d'un « incident critique » dans sa propre classe, pendant la classe d'un collègue, un entretien avec un conseiller d'orientation, une étude de cas en conseil de classe, l'observation attentive et longitudinale d'un élève en difficulté, l'analyse d'un contenu didactique particulièrement difficile à faire passer, etc), bref, une écriture qui d'une certaine façon oblige le stagiaire à s'impliquer, à construire et assumer un point de vue personnel. 
- il s'agit aussi d'une pratique d'écriture dont le but est de construire des habitudes d'écriture de travail (un habitus professionnel) : apprendre à noter les attentes avant le cours, les incidents critiques qui se sont produits, les comportements spécifiques d'élèves, les remarques de collègues, les documents intéressants, les idées qui germent pour élaborer de nouveaux projets etc.

9 Le portfolio de ce point de vue est réflexif car il met en attente. Il permet le retour différé, et ainsi la mise à distance des décisions prises dans le feu de l'intervention, mais aussi des situations et contextes scolaires, des acteurs, des objets travaillés. L'écriture en obligeant à nommer, permet de sortir de la gangue du vécu, des affects et ainsi facilite l'objectivation des situations et le contrôle de soi.

\subsubsection{Le rôle du temps}

10 Le portfolio garde la trace et permet de revenir, il permet la reformulation, (je pensais au début que, maintenant je réalise que...) le classement mais aussi l'effacement, (ça, maintenant je sais).

Il a pour but d'inscrire la formation dans le temps et d'en garder la trace et ce faisant de rendre perceptible l'idée que le temps est un instrument didactique de première importance et que la patience pédagogique est une compétence professionnelle essentielle. Laisser du temps au temps pour laisser aux formés le temps de s'approprier les savoirs, les méthodes, des attitudes intellectuelles, leur laisser le temps d'apprivoiser l'espace dans lequel et par lequel ils apprennent. Pour les stagiaires, commenter en fin d'année le « blason ${ }^{5}$ » qu'ils ont élaboré en début d'année, comparer un début de cours en début et en fin de stage, faire son bilan de formation : les points solides, les savoirs en cours d'acquisition, les questions non résolues mais qui commencent à être mieux formulées, etc, peut contribuer à cette prise de conscience du rôle du temps dans le développement des compétences et de l'identité.

\subsubsection{Un objet où se tissent des connaissances}

11 Les formations et discours que reçoivent les stagiaires sont très éclatés, voire très discordants (voir plus loin). On sait par ailleurs combien le tissage des savoirs acquis par des approches théoriques hors contexte scolaire immédiat et les savoirs acquis, dans et par la pratique ont du mal à se mettre spontanément en réseaux, combien ils ont besoin d'un fort étayage. L'alchimie des premières rencontres avec les élèves, avec les institutions, avec un professeur tuteur servant de "passeur" peut se révéler pour certains la meilleure des formations mais pour d'autres l'occasion de difficultés graves voire de souffrances. Une des raisons d'être des IUFM est ainsi de diversifier précisément les formes d'étayage par lesquelles les savoirs professionnels commencent à prendre forme. Au regard de ces constats, le second postulat que nous défendrons ici est qu'apprendre, se former, donner du sens aux situations de formation se joue dans des processus de tissage que le portfolio favorise et travaille :

- tissage des connaissances entre elles (et elles sont très diverses en formation générale : la violence, l'autorité, des éléments de psychologie, des éléments de réflexion transdidactiques sur la question de la mise en activité des élèves, de l'évaluation, sur la place de l'oral ou de l'écrit pour apprendre, etc.);

- tissage des connaissances avec les savoirs d'action, l'expérience, professionnelle, intellectuelle, affective. 
12 Le portfolio, collection de textes divers (préparations de cours commentées, observation d'élèves, compte-rendu de visites, notes de cours ou de lecture questionnées, etc.) devrait favoriser ce tissage, ces mises en regard a posterioriet ainsi la construction, non de réponses diverses pour «bien faire, bien tenir sa classe », mais l'élaboration progressive de trames conceptuelles complexes, à partir desquelles penser, décider de l'action pédagogique à mener.

\subsection{Le portfolio un mode de socialisation ${ }^{6}$ et de construction identitaire}

\subsubsection{Avec ses pairs}

13 Le troisième postulat mis en œuvre est celui de la construction sociale des savoirs ${ }^{7}$, construction qui accompagne la construction progressive de l'identité professionnelle. Cette co-construction des savoirs et de l'identité professionnelle du stagiaire a lieu d'abord avec ses pairs. Avec et contre eux et leurs regards portés sur lui, le stagiaire construit sa nouvelle identité professionnelle, sa différence, son talent propre. Cette expérience de la différence dans la perception et la résolution d'un problème professionnel, à l'intérieur d'une même discipline, entre les disciplines est l'un des points forts des modules de formation qui rassemblent des stagiaires de plusieurs disciplines, plusieurs niveaux d'enseignement, c'est un des apports fondamentaux de l'analyse de pratiques en groupe de type GEASE.

14 L'écrit permet aussi cette confrontation, oblige ceux qui choisissent habituellement de se taire ou de rester en retrait du débat de s'impliquer et prendre un peu position. On postule donc que c'est parce qu'il est confronté à ses pairs qui vont lire des analyses ou des observations qu'il aura notées dans son portfolio, qui vont discuter son point de vue qu'on peut espérer une implication plus forte de la part du stagiaire. Cette confrontation est tout à fait différente de celle qu'il peut avoir avec un tuteur ou un conseiller qui construit d'autres rapports aux savoirs professionnels, d'autres rapports identitaires. Les autorisations à dire, se dire, penser ne sont pas identiques.

\subsubsection{Un espace possible de dialogue avec les formateurs}

15 La formation des maitres est une formation d'adultes responsables de leurs actes, de leurs jugements, que l'on souhaite rendre maîtres et acteurs de leur parcours de formation. Les PLC2 comme les PE2 sont fréquemment visités et leurs travaux à l'IUFM ou en stage pratique font l'objet de nombreuses évaluations ou rapports, lesquels ont la double caractéristique d'être privés et assez fréquemment contradictoires dans les conseils ou remarques adressés au stagiaire. Cette confrontation du stagiaire à une diversité de points de vue (voir supra)sur sa pratique n'est certainement pas à remettre en question bien au contraire : par là même il prend conscience qu'il n'y a pas dans l'institution une culture professionnelle mais des cultures différentes à partir desquelles il devra se situer. Nous postulons ici que cette confrontation, pour être efficace et non déstabilisante a besoin d'être étayée, discutée, montrée, et d'être l'occasion d'un débat « démocratique ». Ce qui veut concrètement dire que dans le portfolio ou aujourd'hui ce qu'on appelle à l'IUFM de Montpellier le "dossier professionnel», devraient pouvoir figurer les remarques et conseils des divers formateurs, avec lesquels le stagiaire devrait pouvoir argumenter (rien ne l'y oblige, mais rien ne l'interdit). Ces remarques, mises en 
discussion ou simplement lues par des pairs, permettent au stagiaire de prendre du recul, de sortir de l'émotion ou de certitudes trop rapidement construites.

Force est d'admettre qu'un tel dialogue (gage encore une fois de démocratie) nécessite de la part des formateurs d'accepter de jouer le jeu de la confrontation ouverte avec d'autres points de vue, conduit aussi à un certain relativisme des regards (le tuteur de terrain, le PIUMF, le didacticien spécialiste de la discipline, le chef d'établissement, l'inspecteur, le conseiller pédagogique, ne conseillent pas de la même façon...).

Cette mise en débat, publique (à l'intérieur du groupe) des points de vue des formateurs est très riche. Lors de l'expérimentation dans le module français du portfolio, on a pu remarquer combien la discussion écrite que nous tenions à propos des travaux individuels suscitait des réactions et débats divers entre les stagiaires. En dialoguant ainsi à l'écrit avec l'un d'entre eux nous introduisions des discussions en cascades (et ce d'autant plus que nous prenions des positions nettes).

On peut penser que c'est au travers de tels dialogues entre les stagiaires et entre les formateurs (car les portfolios peuvent être lus par divers formateurs) que se construit une communauté et une véritable culture, commune et hétérogène, de la formation.

\subsection{Le portfolio comme mise en cohérence de la formation et de l'évaluation}

\subsubsection{Des ruptures nécessaires dans l'évaluation certificative du côté de l'institution}

19 Qu'on ne se le cache pas non plus, cette manière de penser la formation et ses pratiques d'évaluation certificative va à l'encontre des conceptions et pratiques institutionnellement en vigueur et s'inscrit en rupture avec celles-ci. On évalue jusqu'à présent, dans le cadre des commissions certificatives, le stagiaire non à partir de ses travaux, de ses pratiques, mais à partir des discours sur ses travaux, sur ses pratiques, sur ses attitudes dans l'établissement, etc. (les multiples évaluations et rapports écrits ou oraux des divers formateurs). Le point de vue du stagiaire sur son parcours, ses pratiques en début et en fin d'année, ses réussites, ses difficultés, la démarche réflexive qu'il a pu mettre en place dans la confrontation de son expérience dans la classe et les formations théoriques qu'il a pu recevoir, ne sont pris en compte (un peu) que dans le cadre du mémoire professionnel. La parole propre du stagiaire, globalement, pèse très peu. La pratique du portfolio, comme instrument d'évaluation parmi d'autres, permet que cette parole du stagiaire sur des objets réels de sa formation soit entendue, que sa compétence réflexive soit objet d'évaluation. Le portfolio en représentant son travail et son point de vue sur son travail, le représente et donne des indications sur l'émergence de son identité professionnelle, sur le professeur qu'il a envie d'être.

\subsubsection{Des ruptures nécessaires dans le rapport au savoir des stagiaires}

Mais la démocratie ne se décrète pas, elle s'apprend. Ce n'est pas parce qu'on donne la parole aux stagiaires qu'ils la souhaitent et s'en emparent. La pratique du portfolio est exigeante, elle leur demande d'adopter des comportements non de consommateurs de la formation mais d'assumer en la montrant et la réfléchissant avec leurs formateurs, la responsabilité de leur parcours de formation qui serpente entre la classe, les établissements et les modules de formation à l'IUFM. Il s'agit alors non de renvoyer dos à 
dos les diverses instances de formation, mais d'accepter d'en travailler ensemble les diverses rationalités.

\section{Ce que nous a appris l'expérience et la recherche}

21 L'expérience du portfolio est déjà conséquente : trois ans d'utilisation dans les modules français de Perpignan et 1 an sur quatre groupes de formation générale et commune en PLC2 (2001-2002). De plus, un élément du portfolio, le journal de bord a fait l'objet d'une recherche spécifique (Bucheton J. et Chabanne, 2001), recherche qui nous a amenés à le modifier dans le cadre d'un projet innovant: (Decron, Bucheton, Chabanne, Cailler et Parayre, 2002). C'est à partir de ces savoirs de l'action et de la recherche qu'on voudrait maintenant souligner quelques unes des réussites, difficultés ou dérives auxquelles il faut réfléchir pour que les conditions de réussite du portfolio soient optimum.

\subsection{Définir les objets pouvant donner lieu à écriture ${ }^{8}$}

L'esprit du portfolio est de construire un équilibre entre des objets contraints, proposés par les formateurs et des objets choisis par le stagiaire. On n'est pas ici dans la pédagogie du texte libre ou du journal autobiographique de formation (Quattrevaux, 2002). L'idée est au contraire que pour socialiser les savoirs construits et les évaluer, il faut des éléments de comparaison. En français quatre tâches ont été proposées : une observation d'élèves, une observation d'un maitre (incidents critiques, écarts entre le prévu et le réalisé), des éléments d'un journal de bord et un texte de synthèse sur le stage en responsabilité. En FGC, Une série de tâches plus ouvertes et diverses ont été proposées (le compte-rendu d'une visite en ZEP, une préparation de cours commentée, un entretien avec une personne de son choix sur les questions de citoyenneté, le compte rendu du projet Aide au travail individualisé, une observation d'élèves dans la classe d'un collègue du groupe, un bilan de formation). Les stagiaires devaient choisir un certain nombre de ces « objets", travaillés en module, mais pouvaient aussi ajouter à leur gré des notes de cours, des textes théoriques retenus comme importants, des travaux personnels. Dans leur majorité les portfolios rendus en FGC faisaient de 12 à 30 pages, beaucoup plus en français.

Une analyse a posteriorides objets choisis en FGC révèlent rapidement qu'ils ne sont pas forcément les plus pertinents pour installer la réflexivité professionnelle attendue. Il est difficile pour les stagiaires d'en repérer les complémentarités. La " collection de textes » en FGC est simple juxtaposition là où en français elle construit des cohérences dans les compétences professionnelles en train d'émerger. On n'épiloguera pas longtemps làdessus sinon pour dire que l'expérience montre que le choix des objets mérite une réflexion importante des formateurs. Mieux vaut sans doute moins d'objets mais des objets plusieurs fois repris. Il nous a fallu expérimenter pour l'apprendre. Il est probable aussi que selon les disciplines les « objets" intéressants à passer au crible de l'écriture réflexive ne sont pas forcément les mêmes.

\subsection{La nécessaire clarté du contrat}

Trois ans de bricolage dans le module français ont été nécessaires pour rédiger pour les stagiaires un contrat à peu près correct expliquant les présupposés théoriques du 
dispositif de formation, les consignes précises, les dates de remise des divers éléments du portfolio, et les critères d'évaluation. Pour comprendre aussi que ce portfolio devenait trop lourd à partir du moment ou son principe se généralisait dans d'autres disciplines. Il aura fallu aussi du temps pour disposer de «modèles» permettant au stagiaire de comprendre un peu ce qu'on attendait de lui. Dans le dispositif innovant FGC, nous n'avons pas eu le temps de verbaliser suffisamment le contrat, de l'écrire et le négocier; Nous l'avons inventé, bricolé en avançant ce qui a provoqué chez certains stagiaires un sentiment d'inquiétude et d'insécurité, voire de refus. Son acceptation et surtout son interprétation ont été beaucoup plus difficiles mais surtout très variables d'un groupe à l'autre et très dépendantes du discours du formateur ce que nous n'avons pas observé dans les quatre groupes PE2 de Perpignan.

\subsection{Les dérives formalistes}

Le portfolio n'est pas jugé sur sa forme : l'écriture demandée à la différence du mémoire professionnel est une écriture grise, une écriture de travail à l'exception du dernier document de synthèse où finalement le stagiaire rédige un petit essai de trois ou quatre pages pour tirer les leçons de sa formation. On a affaire là à un texte argumentant, à un commentaire qui renvoie à des formes sociales proches du rapport et que tout enseignant doit être en mesure un jour de rédiger pour faire un bilan de projet, de rencontres, etc.

Pourtant, les dérives scolaires formalistes semblent inéluctables : il est difficile de faire comprendre parfois que le plus important ce n'est pas de recopier ses notes de cours ou ses préparations mais de penser sur, de penser contre, de penser avec ces notes. À décharge de ces stagiaires très "scolaires", il faut admettre que la tradition scolaire et universitaire ne les a guère préparé à une conception de l'écriture, instrument du travail intellectuel. De plus il faut aussi considérer qu'un certain amour du beau texte bien présenté, du travail bien fini, du bel ouvrage explique aussi certaines résistances à livrer cette " écriture de travail». Changer les conceptions comme le rapport à l'écriture du stagiaire, comme des formateurs rencontre nécessairement des résistances qu'il faut mettre en discussion.

\subsection{Pistes pour l'évaluation}

27 Ne nous le cachons pas, les difficultés de l'évaluation de tels objets restent entières et sont à travailler et ajuster.

Suggérons ici simplement quelques pistes ${ }^{9}$ et quelques principes qui pourraient servir de point de départ à une réflexion sur l'évaluation des portfolios, dossiers professionnels et autres écrits de même obédience.

Si on considère qu'on cherche par ce travail d'écriture à développer des attitudes réflexives, l'implication dans la formation, la capacité à avancer dans son projet de formation en se frottant à la parole des autres, à sa propre parole, la capacité à construire un point de vue personnel argumenté sur les problèmes professionnels rencontrés, etc, alors il faut se donner des critères qui rendront visibles ces objectifs. Nous savons bien évaluer les formes des écrits, les manques de ceci ou cela, nous avons beaucoup moins l'habitude de nous intéresser à ce que nous disent les textes des points de vue construits par leurs auteurs ${ }^{10}$. 
29 1. On peut mesurer l'implication et la réflexivité des stagiaires à toute une série de critères y compris formels : les reprises, ratures, brouillonnements, commentaires à des moments différents d'un même texte, mais aussi les formes d'énonciation, de modalisations, de trace des affects, etc. ${ }^{11}$, à des indicateurs fiables d'un travail personnel comme la présence d'exemples précis, situés dans des contextes identifiés.

2. On peut aussi identifier dans les portfolios des différences de centration : sur sa propre parole et présence en classe, sur les savoirs à enseigner, sur les élèves en difficultés, sur les problèmes d'autorité, de relations avec les collègues, sur les attentes du tuteur, de l'établissement, des parents, etc. On peut ainsi repérer pour les réguler et les discuter des «postures d'enseignement » diverses qui peuvent devenir problématiques lorsqu'elles ne bougent pas, ou ne sont pas assez ouvertes à différentes centrations.

31 3. La présence ou non des élèves, vus dans la singularité de leurs réussites ou difficultés, et non comme une entité générale : (la classe, les élèves ont bien compris) est un des indicateurs les plus importants de l'entrée dans la professionnalité enseignante. Ces stagiaires là ont compris qu'on fait classe à des personnes réelles et pas seulement à des sujets « épistémiques ».

32 4. D'une façon générale on constate que les savoirs enseignés ne sont jamais questionnés, ni même problématisés : les stagiaires ne se l'autorisent pas, sans doute est-ce trop tôt. La réflexion sur les programmes, leur évolution, leur transformation nécessaire n'est sans doute pas encore possible.

33 5. Enfin il est clair que pour certains, écrire est considéré comme une perte de temps, que probablement ils apprennent et construisent leurs compétences professionnelles autrement, alors que d'autres s'emparent de l'écriture proposée comme d'un véritable outil pour penser leur formation et se donner des instruments de travail. Cependant, on évalue ici la capacité à entrer, comprendre et accepter une contrainte institutionnelle, négociée et décidée collectivement. Après tout, on demande aux élèves aujourd'hui d'écrire, parler, lire beaucoup pour apprendre. Cet «habitus de travail nouveau» de l'école devrait pouvoir s'appliquer en premier à nos stagiaires à qui on demande d'en faire « l'expérience » quelle que soit leur formation d'origine. Le métier d'enseignant est d'abord un travail intellectuel, avant d'être un travail d'action et d'interactions, les outils du travail « au bureau » ont besoin aussi d'être affûtés.

\section{BIBLIOGRAPHIE}

BUCHETON D. et CHABANNE J.C. : « Les postures d'enseignants débutants : des manières de penser, interprêter, agir dans la classe ", in Actes du colloque DFLM : Les tâches et leurs entours. Neuchâtel (Suisse), 2001.

CHABANNE J.C. et BUCHETON D. : Écrire en ZEP, évaluer autrement les écrits des élèves. Paris, DELAGRAVE - CNDP, 2002 (à paraître).

CHABANNE J.C. et BUCHETON D. : introduction, pp. 1-27 de Parler et écrire pour penser, apprendre et se construire, l'oral et l'écrit réflexif. Paris, PUF, 2002. 
DELAMOTTE R., GIPPET F., JORRO A. et PENLOUP M.C. : Passages à l'écriture, un défi pour les apprenants et les formateurs. Paris, PUF, 2000.

QUATTREVAUX A. : Journaux de formation, analyse de discours et communication orale. Paris, L'Harmattan, 2002.

PAULSON F.L., PAULSON P.R. et MEYER C.A. : « What makes a portfolio a portfolio ? », in Educational leadership, $N^{\circ} 48$, pp. 60-63, 1991.

VANHULLE S. : «Comprendre des parcours d'écriture réflexive : enjeux de formation et de recherche ", in Chabanne J.C. et Bucheton D. (dir) ; Parler et écrire pour penser, apprendre et se construire, l'oral et l'écrit réflexif. 2002, pp. 227-246.

VANHULLES S. : Des savoirs en Je aux savoirs en Jeu. Liège (Belgique), Université de Liège (thèse de doctorat inédite), 2002.

\section{NOTES}

1. D. Bucheton et J.C Chabanne d'abord, rejoints ensuite par J. Cailler, A. Decron et M. Parayre.

2. CHABANNE J.C. et BUCHETON D. (dir) : L'oral et l'écrit réflexif, parler et écrire pour apprendre et se construire. Paris, PUF, 2001.

3. Il s'agit de C. Asdih, B. Bois, D. Bucheton et C. Dupuy.

4. Voir la thèse de doctorat de Sabine Vanhulles : "Des savoirs en Je au savoir en jeu ", 2002, Université de Liège, qui étudie un portfolio de formation mis en place sur un cycle de trois ans de formation dans un module littératie en Belgique.

5. Dispositif fréquemment utilisé en formation pour amener le stagiaire à se présenter aux autres, à identifier ses attentes, ses craintes.

6. C'est évidemment ici tout le paradigme socio-constructiviste vygotskien qui est ici convoqué.

7. On défend ici en formation le principe d'homologie. Les théories de l'apprentissage que nous défendons sont d'autant plus crédibles et appropriées que nous en démontrons la validité et les limites par nos propres dispositifs de formation

8. On postule ici qu'il n'y a pas d'enseignement sans tâches prescrites et que celles-ci jouent un rôle formatif essentiel.

9. Elles proviennent de la recherche sur les journaux de bord, Bucheton, Chabanne, 2001.

10. CHABANNE J.C. et BUCHETON D. : Écrire en ZEP, évaluer autrement les textes des élèves. Paris, Delagrave-CNDP, 2002.

11. Certaines analyses d'E. Nonnon, relatives à l'écriture du mémoire professionnel sont ici très pertinentes.

\section{RÉSUMÉS}

L'usage du portfolio comme outil d'évaluation et de formation est de plus en plus fréquent. Il introduit cependant des ruptures importantes dans les conceptions de l'évaluation et nécessite de faire la clarté sur les enjeux cognitifs, professionnels et institutionnels qu'il travaille. On développe ici l'idée que le portfolio ou les diverses autres formes d'évaluation voisines comme le 
dossier professionnel permet le développement de postures réflexives et favorise les allers et retours entre le travail en stage et le travail à l'IUFM.

The use of the portfolio as an evaluation and training tool is becoming more and more common. This use of the portfolio, however, introduces major breaks with past practices in conceptions on evaluation and therefore requires that one makes clear what it puts at stake, cognitively professionally and institutionally speaking. This article develops the idea that the portfolio or the various other closely related shapes of evaluation such as the professional record, allows the development of reflective standpoints and furthers the bock and forth feedback between work conducted in teacher training internship and work at IUFM (teacher training college).

\section{INDEX}

Mots-clés : écriture de travail, évaluation, identité professionnelle, savoir professionnel Keywords : professional identity, professional knowledge, professional writing

\section{AUTEUR}

\section{DOMINIQUE BUCHETON}

Professeur, sciences du langage, sciences de l'éducation, IUFM de Montpellier, équipe LIRDEFALFA 